\title{
Canards and the effect of apparent disappearance
}

\author{
Sobolev V.A. \\ Samara State Airspace University
}

\begin{abstract}
The aim of the paper is to give examples of apparent disappearance phenomenon which is typical for microbiological systems. It is well known that canards play an important role in the theory of mixed-mode oscillations consisting of large amplitude, and followed by small amplitude oscillations. In a sense we consider limiting cases of mixed-mode oscillations, when large amplitude oscillations are followed by vanishingly small amplitude oscillations.
\end{abstract}

Keywords: apparent disappearance phenomenon, canard, invariant manifold

Citation: Sobolev V.A. Canards and the effect of apparent disappearance. Proceedings of Information Technology and Nanotechnology (ITNT-2015), CEUR Workshop Proceedings, 2015; 1490: 190-197. DOI: 10.18287/16130073-2015-1490-190-197

\section{Introduction}

The aim of the paper is to give illustrative examples of apparent disappearance phenomena which are typical for microbiological systems. Our interest in this topic was prompted by a recent trip to Israel made by the author several years ago. During the visit he was told about a group of Bedouins who, together with camels, were drowned in a flash flood in the desert. The following day he toured an old cemetery in Jerusalem that contained the remains of some English soldiers. The majority of the soldiers had died in a similar flash flooding event. In both cases, the victims had set up camp on a dry river bed. The river bed remained dry for long periods of time throughout the year. However, this normal state disappeared for a few hours when the catastrophic flooding occurred. Similar behaviour occurs in the illustrative model of a hydrodynamical system shown in the figure below, in which the lower reservoir is empty apart from a very short time interval where it is full.

The second reservoir also empties through a siphon. A small inflow causes the water level in the first reservoir to rise slowly until the U-turn in the siphon is reached. The water is then very quickly transferred to the lower reservoir due to the siphoning effect, filling it almost instantly. The second siphon then causes the lower reservoir to empty at an equally fast rate.

Strange as it may seen, the analogous situations appear in systems of diversified natures, such as in the case for "predator-prey" systems in microbiology. In this paper we discuss some examples and mathematical models of the apparent disappearance phenomenon in population dynamics. 


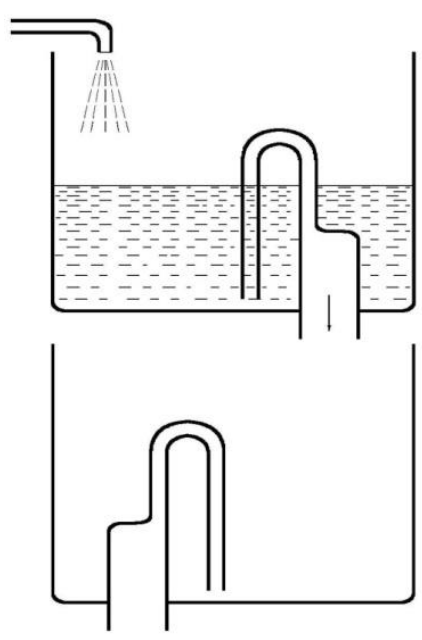

Fig. 1. - Cascade of two Tantal reservoirs

\section{Canards}

To offer a satisfactory mathematical explanation to the apparent disappearance phenomena we will use a technique of singularly perturbed differential equations. Below we will relate the apparent disappearance phenomena to the existence of special kinds of the so called canards or duck--trajectories. These terms have been introduced by French mathematicians [1]. In this section we recall, in a convenient form, the corresponding definitions (see, for example, [2]-[6]).

First we consider a two-dimensional autonomous system:

$\frac{d x}{d t}=f(x, y), \quad \varepsilon \frac{d y}{d t}=g(x, y) y$;

where $x, y$ are scalar functions of time, $\varepsilon$ is a small positive parameter, and $f, g$ are sufficiently smooth scalar functions. The set of points

$S=\{(x, y): y=0\}$

of the phase plane is both a slow curve of the system (1) and a one-dimensional slow invariant manifold. Part of $\mathrm{S}$, all points of which satisfy the inequality

$g(x, 0)<0 \quad(g(x, 0)>0)$,

is called attractive (repulsive). A point $x=A$ of $\mathrm{S}$ is called a turning point if it divides attractive and repulsive parts of $\mathrm{S}$.

We assume that all points at which

$g(x, 0)=0$,

in particular, the turning points, are isolated on $\mathrm{S}$. Let, for simplicity,

$f(A, 0)>0$, and $g(A, 0)<0$, for $x<A$;

$g(A, 0)>0$, for $>A$. 
Trajectories which at first pass along the attractive part of $\mathrm{S}$ and then continue for a while along the repulsive part of S are called canards or duck-trajectories. In the case that a turning point is unique, $\mathrm{S}$ is a canard and it is a longest canard.

Otherwise, this canard may be bounded by neighbouring turning points (or points at which $g(x, 0)=0)$.

In many papers devoted to canards this term is associated with periodic trajectories $[1,4,6]$. In the papers $[7,8]$ it was suggested a canard is a one-dimensional slow invariant manifold if it contains a stable slow invariant manifold and an unstable one, and a canard is obtained as a result of gluing stable (attractive) and unstable (repulsive) slow invariant manifolds at one point of the breakdown surface due to availability of an additional scalar parameter. This approach was proposed for the first time in $[7,8]$ and was then applied to construct canards in $\mathbb{R}^{3}$ [9]-[11], canards for PDE $[8,12]$ and canard travelling waves. Moreover, the use of additional functions instead of additional parameters allowed the construction of black swans [13]-[17], and canard cascades, and the solution of a number of applied problems [18]-[24].

We will introduce some types of canards [25], which are important in the context of this article. Trajectories which at first pass along a repulsive part of $S$ and then continue for a while along an attractive part of $\mathrm{S}$ are called false canards.

In the above cases properties of attraction or repulsion can be viewed as exponential attraction and exponential repulsion by analogy with exponential stability. Using an analogy with the asymptotic stability, we introduce the following notion.

Trajectories which are canards but in which attraction and repulsion are not exponential are called feeble canards.

If on one side of the trajectory it looks like a canard, but on the other side it looks like a false canard, we'll say that this trajectory is a two-faced canard.

It is easy to extend these concepts to the case of vector variables $\mathrm{x}$ and $\mathrm{y}$. If, in particular, $\mathrm{x}$ is a vector and $\mathrm{y}$ is a scalar, then we consider the subspace $y=0$ as a slow manifold. If it contains repulsive and attractive parts, it is called a black swan (attractive/repulsive invariant manifold) [13]-[17].

It should be noted that attractive/repulsive invariant manifolds can be considered for the case of small $\varepsilon$ just as for the case $\varepsilon=1$. The essence of the effect of apparent disappearance is as follows: variable y has vanishingly small amplitude for most of the time. This is due to the fact that trajectories of the system under consideration asymptotically approach an attractive part of the curve (surface) $y=0$ and escape from it repulsive part with a substantial time delay.

\section{Canards' role in modelling the apparent disappearance phenomenon}

Consider the system

$\frac{d x}{d t}=f(x, y), \quad \varepsilon \frac{d y}{d t}=y^{p} g(x, y) ;$

where $p \geq 1, f(x, y)$ is positive for $y<1$ and $f(x, y)<0$ for $y>1$, and moreover, that $\mathrm{f}$ increases to infinity when $x \rightarrow \infty$. Let also $g(x, 0)$ be negative for $x<0$ and positive for $x>0$. Then $y=0$ is a canard; this canard is feeble if $p>1$, and it is a 
two-faced canard if $p=2,4, \ldots$. Suppose that this system has periodic solutions for any small $\varepsilon>0$, and that the graph of this solution crosses the interval $I=\{(x, 1):-\delta<x<0\}$,

where $\delta$ is a positive constant. Then for small $\varepsilon$ the qualitative features of this periodic orbit are predetermined, and may be divided in the six stages.

- S1 The trajectory falls down almost vertically, until it will be of order $\varepsilon \ln \varepsilon$.

- S2 It will continue approach zero moving simultaneously to the right until $x(t)$ becomes equal to 0 . The rate of approaching zero by $y(t)$ is exponential, with an exponent $\sim-1 / \varepsilon$, if $p=1$ and convergence is polynomial if $p>1$.

- S3 The trajectory will still follow very closely the $\mathrm{x}$-axis, but $y(t)$ will increase until it becomes of order $\varepsilon \ln \varepsilon$; this stage is essentially symmetric with $S 2$.

- S4 The trajectory will go up almost vertically, until $y(t)=1$ ("symmetrically" to stage $\mathrm{S} 1$ ).

- S5 The solution will go up (rapidly), simultaneously drifting faster and faster to the left until $x(t)$ is equal to 0 .

- S6 At the final stage the trajectory goes down and to the left until it cross again the interval. This stage is essentially symmetric with Stage S5.

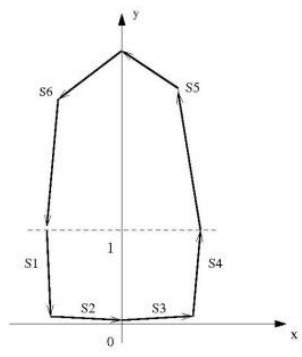

(a)

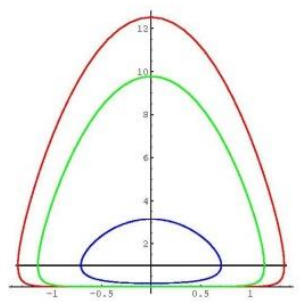

(b)

Fig. 2. - (a): Schematic representation of the structure of periodic trajectories of system (2); (b): Trajectories (4) for $\mathrm{c}=1$ (blue), $\mathrm{c}=1.5$ (green) and $\mathrm{c}=2$ (red).

The dynamics of $y(t)$ exhibits the essential features of theapparent disappearance phenomenon, because all stages, except for S2 and S3, are fast. It is easy to give examples of equations (2) where this scenario happens, that is where the above mentioned periodic trajectories exist. For instance, if $f$ is even in $x$ :

$f(x, y)=f(-x, y)$,

and $g$ is odd:

$g(x, y)=-g(-x, y)$

then any solution is periodic. Figure 2(b), right graphs sample trajectories of an illustrative system

$\frac{d x}{d t}=1-y, \quad \varepsilon \frac{d y}{d t}=x y ;$

Here the trajectories can be written explicitly as 
$x^{2}+2 \varepsilon(y-\ln y)=c$,

where $c>0$ is a parameter.

The crucial question is the following: are there many real world phenomena which are consistent with this scheme, or with its suitable amendments?

The purpose of the paper is to demonstrate that the answer is in the affirmative.

\section{Canards and marine phage population dynamics}

The classic Lotka-Volterra Equations were suggested to model population dynamics of a predator--prey system. They are [26, 27]:

$\frac{d x}{d t}=x(\alpha-\beta y), \quad \frac{d y}{d t}=(-\gamma+\delta x) y ;$

where $x$ and $y$ are functions of time representing the populations of the prey and predator respectively, and $\alpha, \beta, \gamma$, and $\delta$ are positive constants which govern the strength of the interactions between the two species and amongst themselves. This system has a unique positive equilibrium $(\bar{x}, \bar{y})$ when $0=x(\alpha-\beta y), \quad 0=(-\gamma+$ $\delta x) y$; that

$\bar{x}=\gamma / \delta, \quad \bar{y}=\alpha / \beta$.

Hoffmann [27] proposed that for the phage - bacteria interaction the equations be modified such that a power law applies to the Lotka-Volterra equations.

The modified Lotka-Volterra equations are therefore:

$\frac{d B}{d t}=B\left(\alpha-\beta \Phi^{p}\right), \quad \frac{d \Phi}{d t}=\Phi^{p}(-\gamma+\delta B) ;$

we will assume the inequality

$p>1$.

Loosely speaking the equations (6) mean that the "effective size" of the phage population differs from its "physical size", and this effective size is proportional to the power $p$ of the physical size. A naive explanation may be as follows: in the traditional Lotka-Volterra equations (5) and (6), it is assumed that the important meetings are when one predator meets one prey. With the power of two, which was among the best fits in the modified equations, we can hypothesize that unlike the traditional system, it is the meeting of two phages and one bacteria that are important. Correspondingly, the power $\Phi^{p}$ in (6) indicated that that are "hunting-teams" of $p$ phages which are effective. This sounds strange for a fractional $\Phi$, but such ideas are not unusual in mathematics.

This system also has a unique positive equilibrium:

$B_{*}=\frac{\gamma}{\delta}, \quad \Phi_{*}=(\alpha / \beta)^{\frac{1}{p}}$.

Returning to equations (6), we can look for a constant of motion for the system. We do this by dividing the first equation (6) by the second to obtain

$\left(-\gamma \log (B(t)+\delta B(t))+\left(\alpha \Phi(t)^{1-p}\right) /(p-1)+\beta \Phi(t)\right)=$ const.

for any positive solution

$(B(t), \Phi(t))$

of (5). 
Thus,

$W(B, \Phi)=\left(-\gamma \log (B(t)+\delta B(t))+\frac{\left(\alpha \Phi(t)^{1-p}\right)}{p-1}+\beta \Phi(t)\right)$

is a conserved quantity, in other words, the first integral, for the motion of trajectories in this system. The value of the constant is determined by the initial conditions. Rewriting system (6) as

$\frac{d B}{d t}=B\left(\alpha \Phi_{*}^{p}-\Phi^{p}\right), \quad \frac{d \Phi}{d t}=\Phi^{p} \gamma\left(-B_{*}+B\right)$,

we can see the direction vector field in the strictly positive quadrant.

Since

$p>1, \quad \lim W(B, \Phi)=\infty$

as $B \rightarrow 0$, or $\Phi \rightarrow 0$, or $B \rightarrow \infty$, or $\Phi \rightarrow \infty$, the trajectories system could not expand to infinity.

Finally, since the restriction

$W(B)=W\left(B, \Phi_{*}\right)$

is strictly monotone for

$0<B<B_{*}$

we conclude that all trajectories of (6) are periodic, and they orbit anti-clockwise around the equilibrium

$B_{*}=\frac{\gamma}{\delta}, \quad \Phi_{*}=(\alpha / \beta)^{\frac{1}{p}}$.

Let us consider the modified Lotka-Volterra equations (6), when one of the variables $(B$ or $\Phi)$ is significantly faster than the other. We can use singularly perturbed differential systems for modelling such phenomena. The biologically relevant case is "Fast Phages - Slow Bacteria", and the corresponding system is $\frac{d B}{d t}=B\left(\alpha-\beta \Phi^{p+1}\right), \varepsilon \frac{d \Phi}{d t}=\Phi^{p+1}(-\gamma+\delta B)$, with $p>0$.

Proposition 1. The trajectory $\Phi=0$ is a feeble canard with the attractive part $B<\frac{\gamma}{\delta}=B_{*}$ and the repulsive part $B>\frac{\gamma}{\delta}=B_{*}$ for positive $B$ and $\Phi$.

We can observe here the effect of apparent disappearance of phages: they almost disappear for most of the time; however the phages population "explodes" for some short time intervals, see Fig. 3. A curious point is that this feeble canard is two-faced if $p=2,4,6, \ldots$, but that is irrelevant because we are interested in the behavior of our system for positive values of $\Phi$, only.
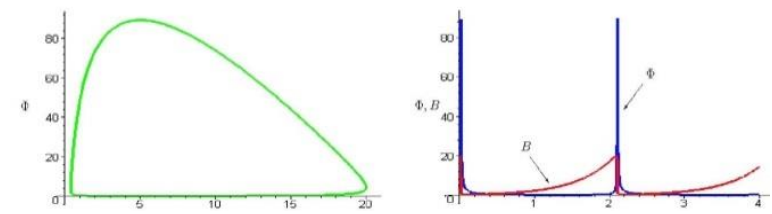

Fig. 3. - Fast phages and slow bacteria model for $\varepsilon=0$., $\alpha=2, \beta=0.1, \gamma=1, \delta=0.2$ 
We consider also the "Fast Bacteria - Slow Phages" case with the corresponding equations

$\varepsilon \frac{d B}{d t}=B\left(\alpha-\beta \Phi^{p+1}\right), \quad \frac{d \Phi}{d t}=\Phi^{p+1}(-\gamma+\delta B)$.

Note that this case is not interesting from the biological point of view.

Proposition 2. The trajectory $B=0$ is a canard with the attractive part $\Phi>$ $\Phi_{*}=(\alpha / \beta)^{\frac{1}{p}}$ and the repulsive part $\Phi<\Phi_{*}=(\alpha / \beta)^{\frac{1}{p}}$.

In this case we observe the effect of the apparent disappearance of bacteria, see Fig. 4.
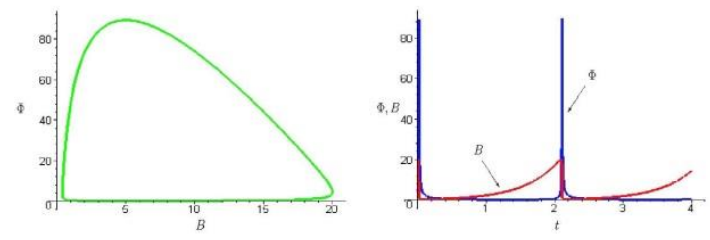

Fig. 4. - Fast bacteria and slow phages model for $\varepsilon=0.2, \alpha=2, \beta=0.1, \gamma=1, \delta=0.2$.

\section{Conclusion}

The modification of the famous Lotka-Volterra equations is considered in the paper. This modification has been recently suggested to model the structure of marine phage populations, who are the most abundant biological entities in the biosphere. The paper attempts to realize the following intentions:

- to make some methodical remarks concerning this modification;

- to discuss new types of canards which arise naturally in this context;

- to present results of some numerical experiments.

\section{Acknowledgements}

This work is supported in part by the Russian Foundation for Basic Research (grants 13-01-97002-p) and the Ministry of education and science of the Russian Federation in the framework of the implementation of Program of increasing the competitiveness of SSAU for 2013-2020 years.

\section{References}

1. Benoit E, Callot JL, Diener F, Diener M. Chasse au canard. Collectanea Mathematica, 1981-1982; 31-32(1-3): 37-119. [in French]

2. Mishchenko EF, Rozov NKh. Differential equations with small parameters and relaxation oscillations. New York: Plenum Press, 1980.

3. O'Malley RE. Singular perturbation methods for ordinary differential equations. Applied Mathematical Sciences, 1991; 89.

4. Mishchenko EF, Kolesov YuS, Kolesov AYu, Rozov NKh. Asymptotic methods in singularly perturbed systems. New York: Plenum Press, 1995. 
5. Vasilieva AB, Butuzov VF, Kalachev LV. The boundary function method for singular perturbation problems. Series in Applied Mathematics, 1995; 14.

6. Arnold VI, Afraimovich VS, Il'yashenko YuS, Shil'nikov LP. Theory of Bifurcations. Dynamical Systems. Encyclopedia of Mathematical Sciences, 1994; 5.

7. Gorelov GN, Sobolev VA. Mathematical modeling of critical phenomena in thermal explosion theory. Combust Flame, 1991; 87: 203-210.

8. Gorelov GN, Sobolev VA. Duck-trajectories in a thermal explosion problem. Applied Mathematics Letters, 1992; 5(6): 3-6.

9. Gol'dshtein V, Zinoviev A, Sobolev V, Shchepakina E. Criterion for thermal explosion with reactant consumption in a dusty gas. Proceedings of the Royal Society of London A, 1996; 452: 2103-2119.

10. Sobolev VA, Shchepakina EA. Duck Trajectories in a problem of combustion theory. Differential Equations, 1996; 32: 1177-1186.

11. Shchepakina EA. Two forms of stability change for integral manifolds. Differential Equations, 2004; 40(5): 766-769.

12. Gorelov GN, Shchepakina EA, Sobolev VA. Canards and critical behavior in autocatalytic combustion models. Journal of Engineering Mathematics, 2006; 56: 143-160.

13. Shchepakina E. Black swans and canards in self-ignition problem. Nonlinear Analysis: Real Word Applications, 2003; 4: 45-50.

14. Shchepakina E, Sobolev V, Mortell MP. Singular perturbations. Introduction to system order reduction methods with applications. Lecture Notes in Mathematics, 2014; 2114.

15. Shchepakina E, Sobolev V. Black swans and canards in laser and combustion models. Singular perturbation and hysteresis, 2005; 207-255.

16. Shchepakina E, Sobolev V. Integral manifolds, canards and black swans. Nonlinear Analysis. Series A: Theory Methods, 2001; 44(7): 897-908.

17. Shchepakina E. Canards and black swans in model of a 3-D autocatalator. Journal of Physics: Conference Series, 2005; 22: 194-207.

18. Sobolev VA, Shchepakina EA. Model reduction and critical phenomena in macrokinetics. Moscow: "Fizmatlit" Publisher, 2010. [in Russian]

19. Shchepakina E. Critical conditions of self-ignition in dusty media. Journal of Advances in Chemical Physics, 2001; 20(7): 3-9.

20. Shchepakina E. Slow integral manifolds with stability change in the case of a fast vector variable. Differential Equations, 2002; 38(10): 1146-1152.

21. Golodova E, Shchepakina E. Maximal combustion temperature estimation. Journal of Physics: Conference Series, 2006; 55: 94-104.

22. Golodova ES, Shchepakina EA. Modeling of Safe Combustion at the Maximum Temperature. Mathematical Models and Computer Simulations, 2009; 1(2): 322-334.

23. Shchepakina E, Korotkova O. Condition for canard explosion in a semiconductor optical amplifier. Journal of the Optical Society of America B, 2011; 28(8): 1988-1993.

24. Shchepakina E, Korotkova O. Canard explosion in chemical and optical systems. Discrete and Continuous Dynamical Systems Series B, 2013; 18(2), 495-512.

25. Gavin C, Pokrovskii A, Prentice M, Sobolev V. Dynamics of a Lotka-Volterra type model with applications to marine phage population dynamics. Journal of Physics: Conference Series, 2006; 55: 80-93.

26. Dancso A, Farkas H, Farkas M, Szabo G. Investigation on a class of generalyzed two-dimensional Lotka-Volterra schemes. Acta Applicandae Mathematica, 1990; 23: 103-127.

27. Hoffmann K H, Rodriguez-Brito B, Breitbart M, Bangor D, Angly F, Felts B, Nulton J, Rohwer F, Salamon P. The structure of marine phage populations. Proceedings of ECOS, 2005; 5-9. 